\title{
Exercício físico para prevenção de quedas: ensaio clínico com idosos institucionalizados em Goiânia, Brasil
}

\author{
Physical exercises to prevent falls: a clinical trial \\ with institutionalized elderly in the city of Goiânia inBrazil
}

Ana Claudia Antonio Maranhão Sá ${ }^{1}$

Maria Márcia Bachion ${ }^{1}$

Ruth Losada de Menezes ${ }^{1}$

${ }^{1}$ Faculdade de Enfermagem, Universidade Federal de Goiás. Rua 227 Qd 68, Setor Universitário. 74605-080 Goiania GO.

ana.claudia.antonio@

terra.com.br
Abstract The scope of this study was to evaluate an intervention program with group physical exercises to prevent falls in the elderly in longterm care institutions. This is a non-randomized clinical trial conducted with 20 institutionalized elderly people in the city of Goiânia in Brazil. The interventions occurred over the period of five months, though the proposed exercise program was based on earlier studies. Standardized measures were used to assess falls, balance and gait, muscle strength, flexibility and fear of falling. After the period of 12 months from the start of intervention there was a significant reduction in the number of falls ( $p=0.046)$. Based on the program, significant differences were observed for point allocation of the maneuvers of balance ( $p=$ 0.001), total scores of the maneuvers of balance and gait ( $p=0.007)$, muscle strength of hand grip $(p=0.001)$ and of lower limbs $(p<0.001)$, flexibility of movement of shoulder flexion $(p=0.001$ ). The intervention using an exercise program proved to be adequate, albeit insufficient to improve the gait, multiple joint flexibility of the spine and hip and fear of falling, or to reduce the number of elderly people who suffered falls from the beginning of the study.

Key words Exercise, Accidental falls, Health of institutionalized elderly people
Resumo Este estudo teve como objetivo avaliar um programa de intervenção com exercícios físicos em grupo na prevenção de quedas em idosos residentes em instituições de longa permanência. Trata-se de um ensaio clínico não randomizado realizado com 20 idosos institucionalizados na cidade de Goiânia, Brasil. As intervenções ocorreram durante cinco meses, sendo que o programa de exercícios proposto foi baseado em estudos anteriores. Medidas padronizadas foram utilizadas para avaliar quedas, equilíbrio e marcha, força muscular, flexibilidade e medo de quedas. A partir do período de 12 meses do início da intervenção houve redução significativa no número de quedas $(p=0,046)$. A partir do programa foram observadas diferenças significantes para pontuação das manobras de equilíbrio $(p=0,001)$, pontuação total das manobras de equilíbrio $e$ marcha $(p=0,007)$, força muscular de preensão palmar $(p=0,001)$ e de membros inferiores $(p<$ $0,001)$, flexibilidade do movimento de flexão dos ombros $(p=0,001)$. A intervenção realizada mediante um programa de exercícios mostrou-se adequada, porém, não suficiente para melhorar a marcha, flexibilidade multiarticular da coluna e quadril e medo de quedas, bem como para reduzir o número de idosos que sofreram quedas a partir do início do estudo.

Palavras-chave Exercício, Acidentes por quedas, Saúde do idoso institucionalizado 


\section{Introdução}

As quedas em idosos constituem-se importante problema de saúde pública em todo o mundo, porque aproximadamente $30 \%$ da população idosa residente na comunidade referem o episódio uma vez ao ano. Esse número sobe para $50 \%$ em pessoas com 80 anos ou mais. Entre os idosos institucionalizados, essa frequência varia entre 30 e $75 \%{ }^{1-3}$.

Quedas devem ser sempre valorizadas, pois são importante causa de mortalidade e morbidade entre idosos, repercutindo em consequências imediatas, como também em efeitos cumulativos e incapacitantes ${ }^{1,3,4}$. Estão associadas a lesões, principalmente fraturas, gerando aumento de despesas com hospitalização e tratamento em longo prazo, e apresentam alto impacto na qualidade de vida ${ }^{4,5}$.

Os profissionais da saúde têm estudado extensivamente para identificar os possíveis fatores de risco modificáveis para quedas em idosos e, assim, planejar intervenções eficazes a serem propostas para a população. Entre os fatores intrínsecos, a literatura atual evidencia que as quedas estão fortemente associadas a déficits de força, flexibilidade, coordenação e equilíbrio. Todos eles apresentam potencial de reversibilidade por meio de exercícios físicos direcionados ${ }^{6,7}$.

Esses exercícios incluem três níveis de abordagem: primário, secundário e terciário. Em sua abordagem primária, a prática regular do exercício físico pode prevenir o surgimento de diferentes doenças e deficiências que podem levar a incapacidades e a maior risco para quedas. No nível secundário, a finalidade é retardar a progressão da deficiência causada pela doença. No nível terciário, o objetivo reside na restauração da funcionalidade para um nível que permita maior autonomia possível no desempenho das atividades cotidianas, para aqueles idosos que tenham atingido um nível de comprometimento que não possa ser revertido8 .

Uma revisão sistemática com meta-análise sobre os efeitos do exercício físico na prevenção de quedas em idosos identificou, como efeito do exercício, a redução em $17 \%$ na taxa de queda9.

No Brasil, os estudos sobre quedas em idosos institucionalizados e não institucionalizados são predominantemente epidemiológicos, em que se buscam os fatores que predispõem essa população ao evento ${ }^{10,11}$. São escassos os ensaios clínicos envolvendo a prevenção de quedas, especialmente com a prática de exercícios físicos ${ }^{12,13}$.

Dessa forma, este estudo teve como objetivo avaliar um programa de intervenção com exercí- cios físicos em grupo na prevenção de quedas em idosos residentes em instituições de longa permanência.

\section{Métodos}

\section{Delineamento}

Foi realizado um ensaio clínico não randomizado com um grupo de 20 idosos institucionalizados.

\section{Participantes e local do estudo}

Foram selecionados idosos de ambos os sexos, com 60 anos ou mais, moradores em duas Instituições de Longa Permanência para Idosos (ILPI) na cidade de Goiânia, Brasil.

Para serem incluídos no estudo, os idosos atenderam aos seguintes critérios: ser morador de ILPI, na modalidade de residências em condomínio; não estar inserido em programa de exercícios físicos regulares; não apresentar déficit cognitivo, auditivo, visual ou motor severamente limitante, atestado por um médico especialista responsável. Dentre os 61 moradores das instituições, 39 atenderam aos critérios de inclusão.

Foram estabelecidos os seguintes critérios de exclusão: deixar de realizar qualquer uma das avaliações propostas durante o seguimento do estudo e faltar a seis sessões de exercícios físicos consecutivas.

Os 39 idosos elegíveis foram convidados a participar do estudo. Destes, cinco recusaramse. Assim, inicialmente, a amostra foi constituída de 34 idosos. No decorrer do estudo foram excluídos 14 participantes: um apresentou declínio cognitivo, atestado pelo médico responsável, que impossibilitou sua permanência no programa de exercícios e 13 desistiram de participar ao longo do seguimento. Assim, a amostra foi constituída de 20 idosos, os quais concluíram todas as avaliações e intervenções previstas.

O estudo foi aprovado pelo Comitê de Ética e Pesquisa da Universidade Federal de Goiás. Todos que aceitaram participar da pesquisa assinaram o Termo de Consentimento Livre e Esclarecido, atendendo à Resolução 196/96 do Conselho Nacional de Saúde. Foi garantido aos participantes anonimato e liberdade de retirar o consentimento a qualquer tempo, sem penalidade alguma.

Todo o programa de intervenção foi conduzido por uma fisioterapeuta e sete estudantes do curso de fisioterapia, devidamente treinados. 


\section{Intervenção}

As intervenções com exercícios físicos foram realizadas em uma sala adaptada dentro das instituições. Os idosos foram orientados quanto ao vestuário, calçados e hidratação durante as atividades. A sala apresentava boa iluminação, ventilação, piso adequado e com fácil acesso à água, preservando-se a segurança e conforto dos participantes. As intervenções ocorreram de fevereiro/2009 a junho/2009, três vezes por semana, por um período de 18 semanas, totalizando 40 sessões de exercícios em grupo, cada uma com duração aproximada de 2 horas, nas quais foram realizados exercícios de aquecimento, força muscular, equilíbrio, flexibilidade e relaxamento.

Todas as sessões foram iniciadas por uma caminhada em superfície plana, com duração de 5 a 10 minutos, com o objetivo de promover o aquecimento. Após descanso de 1 a 3 minutos, foram realizados os exercícios ativos livres e resistidos, no intuito de aumentar a força muscular dos membros superiores, inferiores e tronco e a capacidade de manter ou melhorar o equilíbrio. A duração média dessa etapa foi 60 minutos. A cada série, nesse período, promoveu-se descanso de 1 minuto. $\mathrm{O}$ volume foi de uma a três séries de exercícios, de 8 a 10 repetições, desenvolvidas de forma contínua. Cada repetição deveria ser feita em aproximadamente 7 segundos, sendo 3 segundos para realizar o movimento, 1 segundo mantendo-se a posição alcançada e 3 para retornar à posição inicial. Inicialmente não houve peso extra, além do peso dos próprios membros, que serviram de carga inicial. No começo foi executada apenas uma série por exercício, com oito repetições cada. Após os idosos executarem os exercícios com facilidade, a carga foi escolhida e avaliada pelo fisioterapeuta de maneira que, em cada série, o idoso conseguisse realizar de 8 a 10 repetições. Quando o idoso conseguia realizar mais de 10 , a carga era aumentada.

Em um terceiro momento foram realizados exercícios ativos livres para manter ou aumentar a flexibilidade. A duração média dessa etapa foi 50 minutos, adotando-se descanso de 10 segundos entre as repetições. Cada exercício foi executado três vezes em cada sessão de treinamento. O idoso deveria chegar à posição desejada lentamente, alcançando a maior amplitude de movimento possível, sem que houvesse dor. A posição foi mantida por 10 segundos nas três primeiras sessões e depois passou a 30 segundos até o final do programa. Depois de manter-se na posição, o participante relaxava e voltava lentamente para a posição inicial, terminando, assim, uma repetição.

Ao final de cada sessão era realizado um relaxamento com duração de 10 a 15 minutos. Nesse momento, o idoso escolhia o decúbito de sua preferência, mantendo-se deitado em colchonete. Utilizava-se música suave, para facilitar o procedimento. Concomitantemente, ao longo desse período, os idosos receberam massagem nas costas, membros superiores e inferiores mediante utilização das mãos e bolinhas de látex de cinco centímetros de diâmetro, enquanto eram estimulados por quatro a cinco vezes para inspirar profundamente e expirar três vezes. O Quadro 1 apresenta a descrição dos exercícios.

A elaboração, organização e padronização do programa de exercícios propostos baseou-se em um conjunto de guias e pesquisas ${ }^{6,14-18}$.

\section{Materiais e equipamentos utilizados durante o programa de intervenção}

Foram utilizados colchonetes, travesseiros, cadeiras com assento e encosto, faixas de algodão, halteres, caneleiras, aparelho de som e listas telefônicas (para apoio de cabeça e pés).

\section{Variáveis estudadas}

As variáveis sociodemográficas, condições de saúde-doença e aspectos motores foram avaliadas no início da pesquisa.

A variável queda foi estudada mediante o autorrelato dos idosos participantes, considerando o período de doze meses anteriores ao início das intervenções e o período de doze meses a partir do início da pesquisa. A queda seria considerada quando os idosos relatassem a ocorrência de um evento inesperado e não intencional em que parte do corpo entrasse em contato com o solo ou com algum nível mais baixo que a cintura pélvica.

Esclarecemos que ao término do programa de exercícios os idosos retomaram a rotina anterior, em que não realizavam qualquer tipo de exercício físico.

As variáveis equilíbrio, marcha, força muscular, flexibilidade e eficácia de quedas foram avaliadas na primeira fase da pesquisa (I) anterior ao início do programa de exercícios; na fase (II), após 9 semanas de participação no programa, ocorridas 20 sessões; e na terceira fase (III), ocorridas 40 sessões de exercícios, ao término do programa, ou seja, na $18^{\text {a }}$ semana. 
Quadro 1. Descrição do programa de intervenção com exercícios físicos.

\begin{tabular}{|c|c|}
\hline $\begin{array}{c}\text { Exercício } \\
\text { (duração, frequência, período) }\end{array}$ & Programa (exercícios supervisionados, em grupo) \\
\hline $\begin{array}{l}\text { Aquecimento } \\
\text { (5-10 min., 3x por sem., } 18 \mathrm{sem} .)\end{array}$ & Andar levemente rápido em superfície plana. \\
\hline $\begin{array}{l}\text { Força Muscular e Equilíbrio } \\
\text { (60 min., 3x por sem., } 18 \text { sem.) }\end{array}$ & $\begin{array}{l}11 \text { exercícios } \\
\text { Sentado em uma cadeira: } \\
\text { 1) abdução dos MMSS; } 2 \text { e } 3 \text { ) flexão e extensão do cotovelo } \\
\text { direito e esquerdo; 4) flexão dos ombros; 5) puxada horizontal } \\
\text { com mão em pronação dos MMSS. } \\
\text { Em pé: } \\
\text { 6) flexão plantar dos MMII; 7) flexão de joelho D e E; } 8 \text { e 9) } \\
\text { flexão e extensão de quadril D e E; 10) abdução do quadril D e E; } \\
\text { 11) levantar e sentar da cadeira sem a utilização das mãos. }\end{array}$ \\
\hline $\begin{array}{c}\text { Flexibilidade } \\
\text { (50 min., 3x por sem., } 18 \text { sem.) }\end{array}$ & $\begin{array}{l}13 \text { exercícios } \\
\text { Sentado em uma cadeira: } \\
\text { 1) alongamento da musculatura do punho (flexores do antebraço } \\
\text { e carpo) e musculatura anterior do braço (bíceps braquial); 2) } \\
\text { alongamento da musculatura extensora do punho e carpo; 3) } \\
\text { alongamento da musculatura posterior do braço (tríceps braquial); } \\
\text { 4) alongamento da musculatura rotadora de tronco; 5) } \\
\text { alongamento da musculatura flexora lateral do pescoço. } \\
\text { Decúbito dorsal: } \\
\text { 6) alongamento da musculatura da panturrilha (tríceps sural); 7) } \\
\text { alongamento da musculatura posterior da coxa (isquiotibiais); 8) } \\
\text { alongamento da musculatura anterior da coxa (quadríceps } \\
\text { femoral); } \\
\text { 9) alongamento da musculatura lateral e medial da coxa } \\
\text { (rotadores laterais e mediais); 10) alongamento da musculatura } \\
\text { rotadora de ombro (rotadores mediais e laterais); 11) alongamento } \\
\text { da musculatura rotadora do pescoço; 12) alongamento da } \\
\text { musculatura flexora e extensora do pescoço. } \\
\text { Decúbito lateral: } \\
\text { 13) alongamento da musculatura anterior da coxa (quadríceps } \\
\text { femoral). }\end{array}$ \\
\hline $\begin{array}{c}\text { Relaxamento } \\
(10-15 \mathrm{~min} ., 3 \mathrm{x} \text { por sem., } 18 \mathrm{sem} .)\end{array}$ & $\begin{array}{l}\text { Deitado, decúbito elegido pelo participante: } \\
\text { Respirações e massagens. }\end{array}$ \\
\hline
\end{tabular}

\section{Instrumentos de coleta de dados}

\section{Perfil sociodemográfico, condições} de saúde-doença e aspectos motores

Utilizou-se um formulário padronizado ${ }^{19}$ para guiar a entrevista dos idosos nas próprias residências, que envolveu questões do perfil sociodemográfico, condições de saúde-doença e aspectos motores autorrelatados pelos participantes. Todos os dados foram checados em prontuá- rios arquivados nas instituições e com o grupo de profissionais que atuavam nas instituições (assistente social, psicólogos, educador físico e auxiliar de enfermagem).

\section{Equilíbrio e marcha}

Estas variáveis forma medidas pela escala "Performance - Oriented Mobility Assessment" POMA - Brasil. A escala, desenvolvida em inglês ${ }^{20}$, foi traduzida e adaptada para o português para 
utilização em idosos institucionalizados ${ }^{21}$. É composta de duas avaliações relacionadas ao equilíbrio e à marcha. A "Avaliação do Equilíbrio Orientada pelo Desempenho" pontua o desempenho do paciente ao realizar 13 atividades que reproduzem movimentos cotidianos que requerem equilíbrio. A nota máxima que se pode obter por meio dessa avaliação é 39, e a mínima, 13. Na "Avaliação da Marcha Orientada pelo Desempenho" são observados nove itens por meio da solicitação de deambulação contínua por um trajeto. A pontuação máxima alcançada é 18 , e a mínima, 9 pontos. Os resultados das duas avaliações devem ser somados para obter a nota final. Somatórios mais elevados indicam melhor desempenho.

\section{Força muscular}

A avaliação da força muscular dos membros superiores foi obtida pelo teste da força de preensão manual com dinamômetro hidráulico da marca JAMAR Hydraulic Hand Dynamometer (Model PC-5030J1), respeitando-se o protocolo recomendado pela American Association of Hand Therapists $^{22}$. A força de preensão da mão foi registrada em quilogramas-força. O teste foi realizado três vezes para cada uma das mãos, de forma alternada, iniciando-se com a mão que o sujeito considerasse dominante. A média dentre as três medidas, para cada uma das mãos, foi utilizada como resultado final para a mão correspondente.

A avaliação da força muscular dos membros inferiores foi obtida mediante o "30-s chair stand test”, de se levantar e sentar o número máximo de vezes de uma cadeira em 30 segundos. Este teste tem sido recomendado como uma alternativa prática para medir indiretamente a força dos membros inferiores em idosos devido à correlação moderadamente alta com o teste de 1RM (Repetição máxima) no “leg press", em homens $(0,78)$ e mulheres $(0,71)^{23}$. O resultado é o número total de movimentos completos de se sentar e levantar executados corretamente em 30 segundos.

\section{Flexibilidade}

A amplitude de movimento angular da articulação do ombro foi medida por meio do flexímetro (goniômetro pendular). As medidas foram realizadas de maneira ativa-assistida, ou seja, a cada movimento a ser avaliado, um avaliador demonstrava para o idoso, com movimentos passivos, como deveria percorrer a amplitude de movimento desejada e o idoso realizava de uma a três tentativas, até fazer o movimento correto.
Para a realização das medidas, foram respeitadas as recomendações para aplicação do teste angular descritas por Monteiro ${ }^{24} \mathrm{e}$ as técnicas descritas pela American Academy of Orthopedic Surgeons (AAOS) $)^{25}$.

A flexibilidade multiarticular da coluna e quadril, medida por meio do teste linear Chair Sitand-Reach Test - CSRT (sentar-e-alcançar na cadeira), foi realizada de acordo com o protocolo proposto por Jones et al. ${ }^{26}$. O resultado foi computado como negativo quando o terceiro dedo da mão não tocava a falange distal do hálux da perna em extensão, e positivo quando o referido dedo da mão ultrapassava a falange distal do hálux da perna estendida. No protocolo original, recomenda-se que o teste seja precedido de uma tentativa dos sujeitos para cada um dos membros inferiores com o objetivo de escolher o lado de preferência ou de melhor desempenho.

\section{Escala de eficácia de quedas}

A avaliação da preocupação dos idosos com a possibilidade de quedas durante atividades cotidianas foi procedida mediante a Escala de Eficácia de Quedas - Internacional - Brasil (FES - I Brasil). Conforme recomendações da Prevention of Falls Network Europe (ProFaNE), o instrumento foi traduzido e adaptado culturalmente para a população brasileira ${ }^{27}$. A FES-I apresenta questões sobre a preocupação da possibilidade de cair ao realizar 16 atividades no interior da casa, externas e sociais. Há quatro possibilidades de respostas, que incluem escores de 1 a 4 . O escore total pode variar de 16 a 64 , no qual o valor 16 corresponde à ausência de preocupação e 64, à preocupação extrema em relação às quedas durante a realização das atividades específicas.

\section{Análise estatística}

Realizou-se análise descritiva dos dados de caracterização dos participantes da pesquisa por meio de medidas de frequência, para as variáveis categóricas, e de medidas de posição e variabilidade, para as variáveis quantitativas. A normalidade da distribuição dos dados foi verificada por meio de histogramas e do teste de KolmogorovSmirnov.

A comparação do número de idosos que caíram nos doze meses anteriores à intervenção com o número de idosos que caíram nos doze meses a partir do início desta foi efetuada por meio do teste McNemar. Para comparação entre o número de quedas nos doze meses anteriores e nos doze meses a partir do início das intervenções empregou-se o Teste de Wilcoxon. 
Para análise dos escores do POMA-Brasil, considerando a escala total e subescalas (pontuação parcial do equilíbrio e de marcha) na fase I, II e III da intervenção, utilizou-se o teste de Análise de Variância de um Fator para Medidas Repetidas com Correção, de Bonferroni. A ANOVA para medidas repetidas também foi empregada para análise do desempenho da força muscular dos membros superiores e inferiores dos idosos institucionalizados, ao longo da pesquisa, bem

Tabela 1. Características autorrelatadas do perfil sociodemográfico, condições de saúde-doença e aspectos motores dos idosos institucionalizados $(\mathrm{n}=20)$. Goiânia (GO), 2009.

\begin{tabular}{lcc}
\hline \multicolumn{1}{c}{ Variáveis } & n & $\%$ \\
\hline Perfil sociodemográfico & & \\
Sexo feminino & 17 & 85 \\
Faixa etária de 70 ou mais & 13 & 65 \\
Tempo de institucionalização até 5 anos & 12 & 60 \\
Estado civil sem companheiro & 19 & 95 \\
Condições de saúde-doença & & \\
Percepção subjetiva de saúde ótima ou boa & 18 & 90 \\
Problemas de coluna & 13 & 65 \\
Hipertensão arterial & 09 & 45 \\
Reumatismo & 09 & 45 \\
Ma circulação (varizes) & 08 & 40 \\
Outras doenças & 15 & 75 \\
Uso de medicação regular & 16 & 75 \\
Aspectos motores & & \\
Dificuldade motora em membros superiores & 05 & 25 \\
Dificuldade motora em membros inferiores & 02 & 10 \\
n = número de participantes & & \\
\end{tabular}

como da evolução da flexibilidade do ombro e da pontuação na escala de eficácia de quedas. Para a avaliação da flexibilidade multiarticular da coluna e quadril ao longo da pesquisa, utilizou-se o Teste de Friedman. Quanto ao tratamento estatístico dos dados, foi utilizado o pacote computacional SPSS for Windows (versão 17.0), adotando-se nível de significância menor ou igual $5 \%(\mathrm{p} \leq 0,05)$.

\section{Resultados}

A Tabela 1 apresenta as características autorrelatadas do perfil sociodemográfico, condições de saúde-doença e aspectos motores dos idosos.

Na Tabela 2 pode ser observado que, nos doze meses anteriores à intervenção, o episódio queda foi relatado por 8 idosos, e, a partir do período de doze meses do início da intervenção, o número de idosos que apresentaram relato de queda foi igual a 4. Essa diminuição não foi significativa. Por outro lado, houve relato de 16 quedas no ano anterior à intervenção e somente 05 após o início desta, sendo a diferença significativa.

$\mathrm{Na}$ Tabela 3 encontra-se a comparação do equilíbrio, marcha, força muscular, flexibilidade e medo de quedas nas três fases da intervenção. Foram observadas diferenças significantes para POMA-Equilíbrio, POMA-Total, força muscular de preensão palmar e de membros inferiores e flexibilidade do movimento de flexão dos ombros. Não foram encontradas diferenças significantes para POMA-Marcha, flexibilidade linear (medida pelo teste Chair Sit-and-Reach) e Escala de Eficácia de quedas.

Tabela 2. Ocorrências de quedas em idosos institucionalizados, 12 meses anteriores ao início da intervenção e nos 12 meses a partir do início da intervenção (n = 20). Goiânia (GO), 2009-2010.

\begin{tabular}{|c|c|c|c|c|c|}
\hline \multirow[b]{2}{*}{ Ocorrência de quedas } & \multicolumn{2}{|c|}{$\begin{array}{l}12 \text { meses anteriores ao } \\
\text { início da intervenção }\end{array}$} & \multicolumn{2}{|c|}{$\begin{array}{l}12 \text { meses a partir do } \\
\text { início da intervenção }\end{array}$} & \multirow[b]{2}{*}{$\mathrm{p}$} \\
\hline & $\mathbf{n}$ & $\%$ & $\mathbf{n}$ & $\%$ & \\
\hline \multicolumn{6}{|l|}{ Número de Idosos que caíram } \\
\hline Sim & 08 & 40 & 04 & 20 & $0,289^{*}$ \\
\hline Não & 12 & 60 & 16 & 80 & \\
\hline \multicolumn{6}{|l|}{ Número de quedas } \\
\hline 0 & 12 & 60 & 16 & 80 & $0,046^{* *}$ \\
\hline 1 & 04 & 20 & 03 & 15 & \\
\hline 2 & 01 & 05 & 01 & 05 & \\
\hline 3 & 02 & 10 & 0 & 0 & \\
\hline 4 & 01 & 05 & 0 & 0 & \\
\hline Total & 20 & 100 & 20 & 100 & \\
\hline
\end{tabular}

* Teste de McNemar, , ${ }^{* *}$ Teste de Wilcoxon, $\mathrm{n}$ = número de participantes 
Tabela 3. Comparação das variáveis estudadas dos idosos institucionalizados nas diferentes fases da intervenção (n=20). Goiânia (GO), 2009.

\begin{tabular}{lcccr}
\hline \multicolumn{1}{c}{ Variável } & Fase I & Fase II & Fase III & p $^{*}$ \\
\hline Equilíbrio e Marcha & & & & \\
$\quad$ POMA - Equilíbrio & $35,9 \pm 2,4$ & $37,4 \pm 1,6$ & $36,4 \pm 2,2$ & $\mathbf{0 , 0 0 1}$ \\
$\quad$ POMA - Marcha & $16,8 \pm 2,0$ & $16,9 \pm 1,9$ & $16,9 \pm 2,2$ & 0,811 \\
$\quad$ POMA - Total & $52,7 \pm 3,8$ & $54,2 \pm 3,0$ & $53,3 \pm 4,2$ & $\mathbf{0 , 0 0 7}$ \\
Força Muscular & & & & \\
$\quad$ Força de preensão manual direita & $18,4 \pm 3,2$ & $19,9 \pm 4,6$ & $21,5 \pm 4,9$ & $\mathbf{0 , 0 0 1}$ \\
$\quad$ Força de preensão manual esquerda & $15,9 \pm 3,9$ & $17,8 \pm 3,7$ & $18,0 \pm 3,6$ & $\mathbf{0 , 0 0 3}$ \\
$\quad$ Número de levantar e sentar & $7,5 \pm 2,3$ & $10,6 \pm 1,6$ & $9,6 \pm 1,8$ & $<\mathbf{0 , 0 0 1}$ \\
Flexibilidade & & & & \\
$\quad$ Flexão de ombro direito & $142,4 \pm 25,7$ & $160,9 \pm 17,6$ & $164,4 \pm 10,6$ & $\mathbf{0 , 0 0 1}$ \\
$\quad$ Flexão de ombro esquerdo & $140,2 \pm 25,5$ & $153,9 \pm 17,4$ & $157,7 \pm 18,8$ & $\mathbf{0 , 0 0 9}$ \\
$\quad$ Chair Sit-and-Reach & $-8,8 \pm 10,8$ & $-6,5 \pm 10,9$ & $-5,8 \pm 8,7$ & $0,180^{* *}$ \\
Medo de quedas & & & & \\
$\quad$ Escala de eficácia de quedas & $26,9 \pm 7,9$ & $24,6 \pm 6,8$ & $26,7 \pm 10,9$ & 0,367 \\
\hline
\end{tabular}

"ANOVA para medidas repetidas, ${ }^{* *}$ Teste de Friedman

\section{Discussão}

O presente estudo verificou que um período de 18 semanas de intervenção com combinação de três modalidades de exercícios físicos supervisionados em grupo, com uma frequência de três sessões semanais com duração média de duas horas cada reduziu significativamente o número de quedas entre os participantes. Com o programa de exercicios os idosos caíram menos. Contudo, o programa não reduziu significativamente o número de idosos que caíram. Foram também observados ganhos significantes para variáveis da aptidão física: equilíbrio, força muscular de preensão palmar e de membros inferiores, amplitude de movimento de flexão dos ombros. Não foram encontrados ganhos significantes para variáveis da aptidão física: marcha, flexibilidade multiarticular da coluna e quadril e o medo de quedas.

Cameron et al. ${ }^{28}$ realizaram um estudo de revisão sistemática com objetivo de avaliar a eficácia das diferentes intervenções destinadas a reduzir quedas em idosos que residiam em nursing care facilities. Foram incluídos 11 ensaios clínicos randomizados que avaliaram os efeitos dos exercícios como estratégia de intervenção única. Essa revisão obteve como resultados que: intervenções com uma única modalidade de exercícios em grupo não reduziram significativamente o número de quedas e o número de idosos que caíram; intervenções com combinação de mais modalidades de exercícios em grupo aumentaram de forma significativa o número de quedas e, apesar de não ter havido diferença significativa no número de idosos que caíram, a estimativa não excluiu a possibilidade de que a intervenção pudesse resultar em um aumento de caidores.

Os resultados encontrados por Cameron et al. ${ }^{28}$ diferem dos encontrados em nosso estudo, pois verificamos diferença significativa no número de quedas referidas nos doze meses anteriores ao início da pesquisa em comparação ao número de quedas no período de doze meses a partir do início da intervenção com exercícios. Uma suposta razão para essa divergência pode ser explicada em razão da diversidade da situação de saúde da população envolvida nos estudos da revisão de Cameron et al..$^{28}$, uma vez que os participantes daqueles ensaios clínicos residiam em nursing care facilities e necessitavam de contínuos cuidados pessoais e serviços de reabilitação, geralmente por um período prolongado. Os idosos que residiam nas ILPI, cenários de nosso estudo, moravam em casas dentro da instituição, do tipo condomínio, e realizavam todas as atividades básicas, intermediárias e avançadas da vida diária de forma independente.

Percebemos aqui a importância de definir claramente o objeto de estudo, pois é comum deparamos com pesquisas que envolvem sujeitos de diferentes níveis funcionais em instituições de mesma natureza. Isso dificulta as comparações de resultados entre pesquisas. É necessário descrever com o máximo de detalhes possível o grau de funcionalidade e de saúde dos sujeitos envolvidos. 
Na revisão sistemática realizada por Gillespie et al. ${ }^{29}$ sobre resultados de exercícios na redução de quedas em idosos da comunidade foram encontrados resultados semelhantes aos nossos, ou seja, redução significativa do número de quedas após programa de exercícios. Nessa revisão foram incluídos 43 ensaios clínicos randomizados. Constatou-se que tanto as intervenções em grupo, com combinação de duas ou mais modalidades de exercícios, como intervenções do Tai Chi, que somam força e equilíbrio, ou intervenções com exercícios domiciliares com mais de uma modalidade de exercícios prescritos de forma individual reduziram de forma significativa o número de quedas e o número de idosos que sofreram o evento. Intervenções realizadas somente com uma modalidade de exercícios reduziram de forma significativa apenas o número de quedas, mas não o número de pessoas que caíram.

Ao analisamos o número de idosos que apresentaram quedas a partir do período de doze meses do início da intervenção, não encontramos diminuição significativa. Três fatores podem ser apontados ao depararmos com este resultado:

1) O pequeno tamanho da amostra, com consequente baixo poder estatístico para esse desfecho e a inclusão de idosos com características semelhantes àquelas dos residentes na comunidade: isso também caracterizou os estudos de Shimada et al. ${ }^{30} \mathrm{e}$ Sihoven et al. ${ }^{31}$, que pesquisaram idosos residentes em ILPI. Ambos estudos concluíram que o treino de equilíbrio, marcha e coordenação em esteira reduziu de forma significante o número de quedas, mas não o número de pessoas que caíram. Os autores citados incluíram amostra de 32 e 27 idosos, respectivamente, com níveis funcionais mais elevados, fatores presentes em nosso estudo.

2) A heterogeneidade da amostra para o risco de quedas pode ter influenciado nos resultados. Segundo Rose e Hernandez ${ }^{8}$ em uma intervenção para prevenção de quedas, o tipo e a intensidade do exercício prescrito devem mudar conforme a classificação do risco de queda dos indivíduos. Esses autores identificaram, na sua revisão da literatura, que, para os idosos classificados como "alto risco de quedas", estratégias de intervenções multifatoriais somadas a programas de exercícios personalizados têm mostrado maiores benefícios.

Em nosso estudo, apesar de pequena parte da amostra ser classificada como de alto risco de quedas, utilizamos os exercícios como estratégia de intervenção única para todos os idosos; talvez este fator possa ter contribuído para não encon- trarmos reduções significativas no número de participantes que caíram a partir do período de doze meses do início da intervenção.

3) A possibilidade de o programa de intervenção não ter sido adequado quanto à intensidade, frequência e duração das sessões dos exercícios para os idosos pesquisados é remota.

Sherrington et al. ${ }^{9}$ realizaram um estudo de revisão sistemática e meta-análise com o objetivo de determinar os efeitos dos exercícios para prevenção de quedas em idosos e estabelecer quais características ou componentes dos programas de exercícios estavam associados com a redução dos episódios. Os resultados forneceram fortes evidências de que os programas de exercícios reduzem os índices de quedas nessa faixa etária em $17 \%$. Três características estavam associadas com a eficácia dos programas de exercícios: 1) treino do equilíbrio com desafio progressivo do equilíbrio em pé e apoio mínimo dos membros superiores; 2) dosagens dos exercícios de, no mínimo, 50 horas de treino, com frequência de duas vezes por semana, duração de 1 hora/ sessão, por período mínimo de 25 semanas; e 3) ausência de um programa de caminhada.

Nosso programa de exercícios atendeu à primeira característica integralmente; superou as horas de treino, a frequência e a duração das sessões preconizadas; o período de realização das intervenções foi menor que 25 semanas e foi utilizada uma pequena caminhada para aquecimento. É possível que resultados positivos em relação ao número de caídores tivessem sidos alcançados se o programa fosse realizado por um período mais prolongado.

Contudo, acreditávamos que a maior duração de horas do programa poderia compensar o período menor de semanas de intervenção. Outro ponto a se destacar é que a avaliação de quedas 12 meses após o início do programa de exercícios incluiu o tempo decorrido do encerramento do programa, quando os idosos haviam retomado a rotina anterior sem engajamento em exercícios físicos, o que pode ter influenciado a não redução significativa do número de idosos que caíram.

Estudos demonstram redução considerável de quedas em idosos, mediante programas de exercícios, sem grandes alterações nas medidas do desempenho físico ${ }^{32-35}$. Em contrapartida, há estudos com programas de exercícios que obtiveram alterações em algumas medidas do desempenho físico, porém não alcançaram redução na incidência de quedas ${ }^{36-38}$. Há, também, estudos que não resultaram em alteração algu- 
ma nas medidas do desempenho físico e não encontraram redução nas quedas ${ }^{39,40}$.

Em nosso estudo, o programa de exercícios obteve alterações na maioria das medidas do desempenho físico: POMA-Equilíbrio, POMATotal, força muscular de membros superiores e inferiores e flexibilidade de ombro. Observouse, também, que na avaliação realizada 9 semanas a partir do início dos exercícios, já havia ganhos significativos para as variáveis do desempenho físico e esses ganhos foram mantidos até o término do programa. Embora não tenham ocorrido ganhos significativos na flexibilidade multiarticular de coluna e quadril e na medida da escala de preocupação dos idosos com a possibilidade de quedas, foi possível notar alterações positivas nessas medidas.

\section{Conclusão}

A intervenção realizada mediante um programa de exercícios mostrou-se adequada para melhorar equilíbrio, força muscular de membros inferiores e superiores e flexibilidade de ombro, contribuindo para redução significativa do número de quedas entre os idosos participantes do estudo; porém, não suficiente para melhorar a marcha, flexibilidade multiarticular da coluna e quadril e medo de quedas, bem como para reduzir o número de idosos caidores após o estudo.

Considerando a queda uma síndrome tão complexa, pode-se compreender a magnitude da dificuldade de se encontrar respostas efetivas para sua prevenção, tanto em relação ao número de idosos que sofrem esse evento quanto na frequência dos episódios.

O presente estudo teve limitações que devem ser discutidas: pequeno número da amostra e seleção dos sujeitos de maneira conveniente, o que reduz o poder estatístico dos testes aplicados e limita a possibilidade de generalizações dos resultados.

Nas duas ILPI investigadas o público-alvo para nossa pesquisa foi composto de moradores que viviam em suas próprias casas dentro da instituição, com nível bom de desempenho funcional; porém, não poderem ser considerados como idosos residentes na comunidade. Cada instituição apresentava 30 casas com capacidade para receber moradores individualmente ou com companheiros; contudo, a ocupação, no momento do recrutamento dos sujeitos, era de 61 moradores e somente 39 eram elegíveis para nossa pesquisa, o que tornou a amostra pequena, com limitações para randomização e grupo controle.

Apesar dessas limitações, o estudo apresentou resultados importantes que possibilitam comparações com outros. Com base nos resultados obtidos e na discussão dos achados, esta pesquisa poderá contribuir para estudos futuros, bem como para a atuação de profissionais no desenvolvimento de programas interventivos para prevenção e redução de quedas entre os idosos. Destacamos que a intervenção desenvolvida foi planejada valorizando-se a sua sustentabilidade nas instituições pesquisadas, em termos dos recursos materiais e área física. Contudo, é necessário que haja investimento na obtenção de recursos humanos qualificados para a implementação de intervenções semelhantes nas ILPI.

\section{Colaboradores}

ACAM Sá foi responsável pela concepção, delineamento, redação e revisão do artigo; MM Bachion e RL de Menezes participaram da orientação da pesquisa e revisão do artigo. 


\section{Referências}

1. Gac EH, Marín LPP, Castro HS, Hoyl MT, Valenzuela AE. Caídas en adultos mayores institucionalizados: Descripción y evaluación geriátrica. Rev Méd Chile 2003; 131(8):887-894.

2. Hoffman MT, Bankes PF, Javed A, Selhat M. Decreasing the Incidence of Falls in the Nursing Home in a Cost-Conscious Environment: A Pilot Study. $J$ Am Med Dir Assoc 2003; 4(2):95-97.

3. Menezes RL, Bachion MM. Study of intrinsic risk factors for falls in institutionalized elderly people. Cienc Saude Colet 2008; 13(4):1209-1218.

4. Hanley A, Silke C, Murphy J. Community-based health efforts for prevention of falls in the elderly. Clin Interv Aging 2011; 6:19-25.

5. Minayo MCS, Souza ER, de Paula DR. Systematic review of the Brazilian academic production about external causes and violence against the elderly. Cienc Saude Colet 2010; 15(6):2719-2728.

6. American Geriatrics Society, British Geriatrics Society, and American Academy of Orthopaedic Surgeons Panel on Falls Prevention. Guideline for the prevention of falls in older persons. J Am Geriatr Soc 2001; 49(5):664-672.

7. Rubenstein LZ. Falls in older people: epidemiology, risk factors and strategies for prevention. Age Ageing 2006; 35(Supl. 2):37-41.

8. Rose DJ, Hernandez D. The Role of Exercise in Fall Prevention for Older Adults. Clin Geriatr Med 2010; 26(4):607-631.

9. Sherrington C, Whitney JC, Lord SR, Herbert RD, Cumming RG, Close JCT. Effective Exercise for the Prevention of Falls: A Systematic Review and MetaAnalysis. J Am Geriatr Soc 2008; 56(12):2234-2243.

10. Alvares LM, Costa Lima R, Silva RA. Ocorrência de quedas em idosos residentes em instituições de longa permanência em Pelotas, Rio Grande do Sul, Brasil. Cad Saude Publica 2010; 26(1):31-40.

11. Lojudice DC, Laprega MR, Rodrigues RAP, Júnior ALR. Quedas de idosos institucionalizados: ocorrência e fatores associados. Rev Bras Geriatr Gerontol 2010; 13(3):403-412.

12. Rebelatto JR, Castro AP. Efeito do Programa de Revitalização de Adultos sobre a Ocorrência de quedas dos Participantes. Rev Bras Fisioter 2007; 11(5):383-389.

13. Resende SM, Rassi CM, Viana, FP. Efeitos da hidroterapia na recuperação do equilíbrio e prevenção de quedas em idosas. Rev Bras Fisioter 2008; 12(1): 57-63.
14. National Institute on Aging. Exercise \& Physical Activity. National Institute of Health. Publication No 09-4258, January, 2009. [página na Internet]. [acessado 2012 jul 11]. Disponível em: http:// www.nia.nih.gov/HealthInformation/Publications/ ExerciseGuide/

15. American College of Sports Medicine. The recommended quantity and quality of exercise for developing and mainaining cardiorespiratory and muscular fitness and flexibility in healthy adults. Position Stand. Med Sci Sports Exerc 1998; 30(6):975-991.

16. Nóbrega ACL, Freitas EV, Oliveira MAB, Leitão MB, Lazzoli JK, Nahas RM, Baptista CAS, Drummond FA, Rezende L, Pereira J, Pinto M, Radominski RB, Leite N, Thiele ES, Hernandez AJ, Araújo CGSA, Teixeira JAC, Carvalho T, Borges SF, Rose EH. Posicionamento oficial da Sociedade Brasileira de Medicina do Esporte e da Sociedade Brasileira de Geriatria e Gerontologia: Atividade física e saúde no idoso. Rev Bras Med Esporte 1999; 5(6):207-211.

17. Nelson ME, Rejeski WJ, Blair SN, Ducan PW, Judge JO, King AC, Macera CA, Castaneda-Sceppa C. Physical Activity and Public Health in Older Adults: Recommendation from the American College of Sports Medicine and the American Heart Association. Med Sci Sports Exerc 2007; 39(8):1435-1445.

18. American College of Sports Medicine. Position Stand. Exercise and physical activity for older adults. Med Sci Sports Exerc 1998; 30(60):992-1008.

19. Menezes RL. Quedas em idosos institucionalizados no município de Goiânia [dissertação]. Goiânia (GO): Universidade Federal de Goiás; 2005.

20. Tinetti ME. Performance-oriented assessment of mobility problems in elderly patients. J Am Geriatr Soc 1986; 34(2):119-126.

21. Gomes GC. Tradução, adaptação transcultural e exame das propriedades de medida da escala "performance - oriented mobility assessment" (POMA) para uma amostragem de idosos brasileiros institucionalizado [dissertação]. Campinas (SP): Universidade Estadual de Campinas; 2003.

22. Balogum JA, Akomolafe CT, Amusa LO. Grip strength: effects of testing posture and elbow position. Arch Phys Med Rehabil 1991; 72(5):280-283.

23. Rikli RE, Jones, CJ. A 30-s chair stand test as a measure of lower body strength in community-residing older adults. Res Q Exerc Sport 1999; 70(2):113-119. 
24. Monteiro GA. Avaliação Angular da Flexibilidade. In: Matsudo SMM, organizador. Avaliação do idoso: física \& funcional. Londrina: Midiograf; 2005.

25. American Academy of Orthopaedic Surgeons (AAOS). Joint motions: method of measuring and recording. Chicago: AAOS; 1965.

26. Jones CJ, Rikli RC, Max J, Noffal G. The reliability and validity of a chair sit-and-reach test as a measure of hamstring flexibility in older adults. Res $Q$ Exerc Sport 1998; 69(4):338-346.

27. Camargos FFO. Adaptação Transcultural e Avaliação das Propriedades Psicométricas da Falls Efficacy Scale - International: Um instrumento para avaliar medo de cair em idosos [dissertação]. Belo Horizonte (MG): Universidade Federal de Minas Gerais; 2007.

28. Cameron ID, Murray GR, Gillespie LD, Robertson MC, Hill KD, Cumming RG, Kerse N. Interventions for preventing falls in older peolple in nursing care facilities and hospitals. Cocharane Database Syst Rev 2010; (7):CD005465.

29. Gillespie LD, Robertson MC, Gillespie, WJ, Lamb SE, Gates S, Cumming RG, Rowe BH. Interventions for preventing falls in older people living in the community. Cochrane Database Syst Rev 2009; (2): CD007146.

30. Shimada H, Obuchi S, Furuna T, Suzuki T. New Intervention Program for Preventing Falls Among Frail Elderly People: The effects of perturbed walking exercise using a bilateral separated treadmill. Am J Phys Med Rehabil 2004; 83(7):493-499.

31. Sihvonen S, Sipila S, Taskinen S, Era P. Fall Incidence in Frail Older Women after Individualized Visual Feedback-Based Balance Training. Gerontology 2004; 50(6):411-416.

32. Barnett A, Smith B, Lord SR, Williams M, Baumand A. Community-based group exercise improves balance and reduces falls in at-risk older people: a randomized controlled trial. Age Ageing 2003; 32(4): 407-414.

33. Lord SR, Castell S, Corcoran J, Dip G, Dayhew J, Matters B, Shan A, Williams P. The Effect of Group Exercise on Physical Functioning and Falls in Frail Older People Living in Retirement Villages: A Randomized, Controlled Trial. JAGS 2003; 51(12):16851692.

34. Faber MJ, Bosscher RJ, Paw MJCA Wieringen PC van. Effects of Exercise Programs on Falls and Mobility in Frail and Pre-Frail Older Adults: A Multicenter Randomized Controlled Trial. Arch Phys Med Rehabil 2006; 87(7):885-896.
35. Freiberger E, Menz HB, Abu-Omar K, Rutten A. Preventing Falls in Physically Active CommunityDwelling Older People: A Comparison of Two Intervention Techniques. Gerontology 2007; 53(5):298-305.

36. Hauer K, Rost, B, Rütscble K, Opitz H, Specht N, Bärtsch P, Oster P, Schlierf G. Exercise Training for Rehabilitation and Secondary Prevention of Falls in Geriatric Patients with a History of Injurious Falls. JAGS 2001; 49(1):10-20.

37. Choi JH, Moon JS, Song R. Effects of Sun-style Tai Chi exercise on physical fitness and fall prevention in fall-prone older adults. J Adv Nurs 2005; 51(2):150157.

38. Shigematsu R, Okura T, Nakagaichi, Tanaka K, Sakai T, Kitazumi S, Rantanen T. Square-Stepping Exercise and Fall Risk Factors in Older Adults: A Single-Blind, Randomized Controlled Trial. J Gerontol A Biol Sci Med Sci 2008; 63(1):76-82.

39. Nowalk MP, Prendergast JM, Bayles CM, D‘Amico FJ, Colvin GC. A Randomized Trial of Exercise Programs Among Older Individuals Living in Two Long-Term Care Facilities: The Falls FREE Program. JAGS 2001; 49(7):859-865.

40. Haines TP, Russell T, Brauer SG, Erwin S, Lane P, Urry S, Jasiewicz J, Condie P. Effectiveness of a video-based exercise programme to reduce falls and improve health-relate quality of life among older adults discharged from hospital: a pilot randomized controlled trial. Clin Rehabil 2009; 23(11):973-985.

Artigo apresentado em 28/07/2011

Aprovado em 29/09/2011

Versão final apresentada em 10/10/2011 